PROCEEDINGS OF THE

AMERICAN MATHEMATICAL SOCIETY

Volume 127, Number 10, Pages 2829-2839

S 0002-9939(99)04825-X

Article electronically published on April 23, 1999

\title{
PI-ENVELOPES OF LIE SUPERALGEBRAS
}

\author{
YURI BAHTURIN AND SUSAN MONTGOMERY
}

(Communicated by Lance W. Small)

\begin{abstract}
In this paper we find necessary and sufficient conditions on a finitedimensional Lie superalgebra under which any associative PI-envelope of $L$ is finite-dimensional. We also extend M. Scheunert's result which enables one to pass from color Lie superalgebras to the ordinary ones, to the case of gradings by an arbitrary abelian group.
\end{abstract}

\section{INTRODUCTION}

In this paper we seek to find what associative polynomial identity algebras can be generated by finite-dimensional Lie superalgebras. To be precise, we consider Lie superalgebras over a field $k$ of characteristic 0 , graded by a finite abelian group $G$, of the form

$$
L=\bigoplus_{g \in G} L_{g}
$$

$L$ has a bracket operation [, ] satisfying the generalized anticommutativity and Jacobi identities:

$$
\begin{gathered}
{[x, y]+\varepsilon(x, y)[y, x]=0} \\
{[[x, y], z]=[x,[y, z]]-\varepsilon(x, y)[y,[x, z]]}
\end{gathered}
$$

for any homogeneous $x \in L_{g}, y \in L_{h}, z \in L_{k}$. Here $\varepsilon: G \times G \rightarrow k^{*}$ is the commutation factor, i.e. a function satisfying

$$
\varepsilon(g, h+k)=\varepsilon(g, h) \varepsilon(g, k), \quad \varepsilon(g, h) \varepsilon(h, g)=1 .
$$

We write $\varepsilon(x, y)=\varepsilon(g, h)$ if $x \in L_{g}, y \in L_{h}$. If $G=\{0\}$ we have ordinary Lie algebras, while if $G=\mathbb{Z}_{2}=\{0,1\}$ and $\varepsilon(1,1)=-1$ we have Lie superalgebras. It is easy to see that $\varepsilon(g, g)= \pm 1$ for any $g \in G$. We set $G_{ \pm}=\{g \in G \mid \varepsilon(g, g)=$ $\pm 1\}$. We call the Lie superalgebras just defined $(G, \varepsilon)$-Lie algebras. For details see [BMPZ ]. If $A=\bigoplus_{g \in G} A_{g}$ is a $G$-graded associative algebra, then setting

$$
[x, y]=x y-\varepsilon(x, y) y x
$$

for $x \in A_{g}, y \in A_{h}$, we make $A$ into a $(G, \varepsilon)$-Lie superalgebra $[A]_{\varepsilon}$ or simply $[A]$.

PI-envelopes of ordinary Lie algebras were studied in [Ba 85]; here we look at the analogous situation for Lie superalgebras. We call a $G$-graded associative algebra $A$ an envelope of $L$ if $L \subset[A]$ and $A$ is generated by $L$. We say that $A$ is a PI-envelope

Received by the editors May 27, 1997 and, in revised form, December 11, 1997.

1991 Mathematics Subject Classification. Primary 17A70, 16W50.

The authors were supported by NSF grant DMS-9500649.

(C)1999 American Mathematical Society 
of $L$ if $A$ is an envelope of $L$ and $A$ satisfies a non-trivial polynomial identity. A related notion is that of special Lie superalgebras: any Lie superalgebra having a PI-envelope is called special. Obviously, by Ado's Theorem [S79a] any finitedimensional Lie superalgebra is special. The universal enveloping algebra $U(L)$ is the most natural example of an envelope for $L$ and any envelope is a homomorphic image of $U(L)$. It is shown in [BMPZ] that if char $k=p>0$, then $U(L)$ is a PI-envelope of $L$ and so in this case the PI-envelope does not have to be finitedimensional. Thus if we are interested in those $L$ for which any PI-envelope is finite-dimensional, then it is natural to restrict to the case char $k=0$. So from now on we assume char $k=0$. In this case if we set $L_{+}=\bigoplus_{g \in G_{+}} L_{g}, L_{-}=\bigoplus_{g \in G_{-}} L_{g}$ and $\operatorname{dim} L<\infty$, then $U(L)$ is a PI-algebra iff $L_{+}$is abelian, i.e. $\left[L_{+}, L_{+}\right]=\{0\}$.

A surprisingly sharp result was obtained in [Ba 85].

Theorem 1.1. Let $L$ be a finite-dimensional semisimple Lie algebra over a field $k$ of characteristic 0 , and let $A$ be a PI-envelope of L. Then $A$ is finite-dimensional and semisimple.

We will give some alternate approaches to Theorem 1.1 in Remark 4.3. A less sharp but more general version of this is the following [Ba 85].

Theorem 1.2. Let $L$ be a finite-dimensional perfect Lie algebra (that is, $L=$ $[L, L])$ over a field $k$ of characteristic zero. Then any PI-envelope $A$ of $L$ is finitedimensional.

These theorems were an important tool used by Yuly Billig [Bi] to prove that no affine Kac-Moody Lie algebra is special (this gave a solution to a well-known problem of Latyshev about the speciality of a homomorphic image of a special Lie algebra).

Our main aim is to generalize Theorem 1.2 to the case of $(G, \varepsilon)$-Lie-superalgebras. If $G \neq G_{+}$, that is, if we have odd elements, we exhibit a whole series of examples of simple Lie superalgebras $s l(n, m), n \neq m, n, m \geq 1$, and $W(n), n \geq 2$, whose PIenvelopes can be infinite-dimensional. So it is only natural that we assume quite often that $G=G_{+}$(the case of so called color Lie algebras). In this case we are able to prove full analogues of Theorems 1.1 and 1.2.

We define a semisimple $(G, \varepsilon)$-Lie-superalgebra as one without solvable $G$-graded ideals. Our results are as follows. We first consider the case when $G=G_{+}$.

Theorem 1.3. Let $L$ be a semisimple $(G, \varepsilon)$-Lie-superalgebra over a field $k$ of characteristic zero with $G=G_{+}$and let $A$ be a PI-envelope of $L$. Then $A$ is finitedimensional and semisimple.

Theorem 1.4. Let $L$ be a $(G, \varepsilon)$-Lie-superalgebra over a field $k$ of characteristic zero with $G=G_{+}, A$ a PI-envelope of $L$. Then $A$ is finite-dimensional if and only if $L$ is perfect (that is, $L=[L, L]$ ).

The case of simple Lie superalgebras different from $s l(n, m)$ and $W(n)$ is not entirely hopeless and we are able to prove the following:

Theorem 1.5. Let $L$ be a simple Lie superalgebra over an algebraically closed field of characteristic zero, different from $\operatorname{sl}(n, m), n \neq m, n, m \geq 1$ and $W(n), n \geq 1$. If $A$ is a PI-envelope of $L$, then $\operatorname{dim} A<\infty$.

In the general case, our final result is as follows. 
Theorem 1.6. Let $L=L_{+} \oplus L_{-}$be a finite-dimensional $(G, \varepsilon)$-Lie-superalgebra over any field $k$ of characteristic zero. Then the following are equivalent:

1. Any PI-envelope of $L$ is finite-dimensional;

2. Any (graded) PI-homomorphic image of $U(L)$ is finite-dimensional;

3. $L_{+}$is a perfect color Lie algebra.

Except in Section 2, we assume that $k$ has characteristic 0.

\section{SChEunERT'S TRICK AND SOME APPLICATIONS}

We will need later the following version of the Poincaré-Birkhoff-Witt (PBW) Theorem.

Theorem 2.1 ([BMPZ ]). Let $L=L_{+} \oplus L_{-}$be a $(G, \varepsilon)$-Lie-superalgebra, $S\left(L_{+}\right)$ the symmetric algebra of the vector space $L_{+}$and $\Lambda\left(L_{-}\right)$the Grassmann algebra of the vector space $L_{-}$. Then $U\left(L_{+}\right)$, the universal enveloping algebra of $L_{+}$, is an associative subalgebra of $U(L)$ generated by $L_{+}$and we have the vector space isomorphisms

$$
U\left(L_{+}\right) \cong S\left(L_{+}\right) \text {and } U(L)=U\left(L_{+}\right) \otimes \Lambda\left(L_{-}\right) .
$$

In the remaining part of this section we consider M. Scheunert's approach enabling one to pass from $(G, \varepsilon)$-Lie-superalgebras to ordinary Lie superalgebras with a number of properties preserved. If $\sigma \in Z^{2}\left(G, k^{*}\right)$ is a multiplicative 2-cocycle, and $L$ a $(G, \varepsilon)$-Lie-superalgebra satisfying (2), (3) with commutation factor $\varepsilon$, then replacing multiplication in $L$ on homogeneous elements $x, y \in L$ by

$$
[x, y]^{\sigma}=\sigma(x, y)[x, y]
$$

we arrive at a $\left(G, \varepsilon^{\prime}\right)$-Lie-superalgebra $L^{\sigma}$ satisfying (2), (3) with commutation factor $\varepsilon^{\prime}=\varepsilon \delta$, where $\delta(x, y)=\sigma(x, y) / \sigma(y, x)$. Let $\varepsilon_{0}: G \times G \rightarrow k^{*}$ be the ordinary superalgebra commutation factor, i.e. $\varepsilon_{0}(g, h)=1$ except $\varepsilon_{0}(g, h)=-1$ for $g, h \in G_{-}$.

Theorem 2.2 ([S79a]). Let $G$ be a finitely generated abelian group, and $\varepsilon: G \times$ $G \rightarrow k^{*}$ a commutation factor with $G=G_{+}$. Then there exists a 2-cocycle $\sigma \in$ $Z^{2}\left(G, k^{*}\right)$ such that $\varepsilon \delta=1$, where $\delta$ is as above.

In other words if $L$ is a color Lie algebra, then $L^{\sigma}$ is an ordinary Lie algebra (with the $G$-grading still preserved!). In the general case when $G \neq G_{+}$note that $\varepsilon^{\prime}=\varepsilon \varepsilon_{0}$ satisfies the conditions of the theorem. Thus we can find $\sigma$ with $\varepsilon \varepsilon_{0} \delta=1$, whence $\varepsilon \delta=\varepsilon_{0}^{-1}=\varepsilon_{0}$. It follows that from any $(G, \varepsilon)$-Lie-superalgebra by a change of the form (7) we can switch to an ordinary Lie superalgebra $L^{\sigma}=L_{0}^{\sigma} \oplus L_{1}^{\sigma}$ with $L_{0}^{\sigma}=L_{+}, L_{1}^{\sigma}=L_{-}$.

Scheunert also shows that we can pass from a $(G, \varepsilon)$-Lie-superalgebra to an ordinary Lie superalgebra by tensoring $L$ with a twisted group algebra $k_{\sigma} G$ ( $\sigma$ as before). Here we give another approach (Proposition 2.4) to this switch which is more appropriate to our main aim. Along the way, we generalize Theorem 2.2 to arbitrary abelian groups (this was done independently by $\mathrm{H}$. Pop [Po] in the case of algebraically closed fields).

Theorem 2.3. Let $G$ be an arbitrary abelian group, and $k$ an arbitrary commutative ring with 1 and with group of units $k^{*}$. Then for any commutation factor $\varepsilon$ : $G \times G \rightarrow k^{*}$ there exists $\sigma \in Z^{2}\left(G, k^{*}\right)$ such that if we set $\delta(g, h)=\sigma(g, h) / \sigma(h, g)$, then $\varepsilon \delta=\varepsilon_{0}$. 
Proof. By the above remarks, it suffices to prove our statement when $G=G_{+}$. In this case we have to find a 2-cocycle $\sigma$ with $\sigma(g, h) / \sigma(h, g)=\delta(g, h)$ for all $g, h \in G$ where $\delta$ is the commutation factor $\delta=\varepsilon^{-1}$. We recall that $\sigma$ is a 2-cocycle with values in $k^{*}$ if for all $x, y, z \in G$ we have

$$
\sigma(x, y) \sigma(x+y, z)=\sigma(x, y+z) \sigma(y, z) .
$$

Let $\mathcal{S}$ be the partially ordered set of all pairs $(H, \sigma), H$ a subgroup of $G, \sigma \in$ $Z^{2}\left(H, k^{*}\right)$ with $\varepsilon \delta=1, \delta$ as before, with $(H, \sigma) \leq\left(H^{\prime}, \sigma^{\prime}\right)$ iff $H \subset H^{\prime}$ and $\left.\sigma^{\prime}\right|_{H}=$ $\sigma$. We can apply Zorn's Lemma to $\mathcal{S}$ because it is obvious that it is non-empty and there is a maximal element for any chain $\left\{\left(M_{\alpha}, \sigma_{\alpha}\right)\right\}$, namely $(M, \sigma)$, where $M=\bigcup_{\alpha} M_{\alpha}$ and $\sigma(g, h)=\sigma_{\alpha}(g, h)$ if $g, h \in M_{\alpha}$. Then we have a maximal element $\left(B, \sigma_{0}\right)$ in $\mathcal{S}$. If $G=B$, we are done. Otherwise there is $a \in G \backslash B$. We set $A=\langle a, B\rangle$. If $\langle a\rangle \cap B=\{0\}$, then for arbitrary $u=s_{1} a+b_{1}, v=s_{2} a+b_{2} \in A$, $s_{1}, s_{2} \in \mathbb{Z}, b_{1}, b_{2} \in B$ we set (following Scheunert)

$$
\sigma\left(s_{1} a+b_{1}, s_{2} a+b_{2}\right)=\delta\left(b_{1}, a\right)^{s_{2}} \sigma_{0}\left(b_{1}, b_{2}\right) .
$$

Then

$$
\sigma \in Z^{2}\left(A, k^{*}\right), \delta(u, v)=\sigma(u, v) \sigma(v, u)^{-1} \text { and }\left.\sigma\right|_{B \times B}=\sigma_{0} .
$$

This contradicts the maximality of $\left(B, \sigma_{0}\right)$. The verification of this will be included while considering the more general case $\langle a\rangle \cap B \neq\{0\}$ and so we pass to this case.

Thus we may assume that $c=p a \in B$ for a suitable prime number $p$. Any element in $A$ can be uniquely written in the form $s a+b, 0 \leq s<p, b \in B$. Also, for brevity, we write $\sigma$ in place of $\sigma_{0}$.

For $u=s_{1} a+b_{1}, v=s_{2} a+b_{2}, s_{1}$ and $s_{2}$ as $s$ just above, $b_{1}, b_{2} \in B$ and $s_{1}+s_{2}=p q+r, 0 \leq r<p$ we set

$$
\sigma(u, v)=\delta\left(b_{1}, a\right)^{s_{2}} \sigma\left(c, b_{1}+b_{2}\right)^{q} \sigma\left(b_{1}, b_{2}\right) .
$$

It is easily seen that our formula extends $\sigma$ to $A$ and

$$
\begin{aligned}
& \sigma(u, v) \sigma(v, u)^{-1} \\
& \quad=\delta\left(b_{1}, a\right)^{s_{2}} \sigma\left(c, b_{1}+b_{2}\right)^{q} \sigma\left(b_{1}, b_{2}\right) \delta\left(b_{2}, a\right)^{-s_{1}} \sigma\left(c, b_{1}+b_{2}\right)^{-q} \sigma\left(b_{2}, b_{1}\right)^{-1} \\
& \quad=\delta\left(s_{2} b_{1}-s_{1} b_{2}, a\right) \delta\left(b_{1}, b_{2}\right)=\delta\left(s_{1} a+b_{1}, s_{2} a+b_{2}\right)=\delta(u, v),
\end{aligned}
$$

following from $\delta(g, g)=\varepsilon(g, g)=1$ for all $g \in G$. It remains to check the cocycle identity (8) for $\sigma$. This will be done by direct verification. Since (9) depends on whether the values of $s_{1}+s_{2}$, etc. are greater than $p$, we have to deal with six cases as described below. Let $X$ denote the left side of (8) and $Y$ the right. If

$$
x=s_{1} a+b_{1}, y=s_{2} a+b_{2}, z=s_{3} a+b_{3},
$$

then

$$
X=\sigma\left(s_{1} a+b_{1}, s_{2} a+b_{2}\right) \sigma\left(\left(s_{1}+s_{2}\right) a+\left(b_{1}+b_{2}\right), s_{3} a+b_{3}\right)
$$

and

$$
Y=\sigma\left(s_{1} a+b_{1},\left(s_{2}+s_{3}\right) a+\left(b_{2}+b_{3}\right)\right) \sigma\left(s_{2}+b_{2}, s_{3} a+b_{3}\right) .
$$

Notice that the " $\delta$-factors" on both sides of $X=Y$ are always the same, namely

$$
\delta\left(b_{1}, a\right)^{s_{2}} \delta\left(b_{1}+b_{2}, a\right)^{s_{3}}=\delta\left(b_{1}, a\right)^{s_{2}+s_{3}} \delta\left(b_{2}, a\right)^{s_{3}}
$$

in all cases except when $s_{2}+s_{3}=p+t, t \geq 0$. In this latter case we have on the right side

$$
\delta\left(b_{1}, a\right)^{t} \delta\left(b_{2}, a\right)^{s_{3}}=\delta\left(b_{1}, a\right)^{s_{2}+s_{3}-p} \delta\left(b_{2}, a\right)^{s_{3}}=\delta\left(b_{1}, a\right)^{s_{2}+s_{3}} \delta\left(b_{2}, a\right)^{s_{3}} \delta\left(p a, b_{1}\right) .
$$


So in this case we have to multiply the right side by $\delta\left(c, b_{1}\right)$ or, alternatively, the left side by $\sigma\left(b_{1}, c\right)$ and the right one by $\sigma\left(c, b_{1}\right)$ after we cancel out the " $\delta$-factors". With this in mind we do not write the " $\delta$-factors" in what follows and we denote by $\tilde{X}$ and $\tilde{Y}$ the remaining portions of the sides. So we pass to considering all possible cases, six in totality.

1. $s_{1}+s_{2}+s_{3}<p$.

$$
\tilde{X}=\sigma\left(b_{1}, b_{2}\right) \sigma\left(b_{1}+b_{2}, b_{3}\right)=\sigma\left(b_{1}, b_{2}+b_{3}\right) \sigma\left(b_{2}, b_{3}\right)=\tilde{Y} .
$$

2. $s_{1}+s_{2}=p+r, s_{2}+s_{3}<p$ (hence $r+s_{3}<p$ ).

$$
\begin{aligned}
\tilde{X} & =\sigma\left(c, b_{1}+b_{2}\right) \sigma\left(b_{1}, b_{2}\right) \sigma\left(c+b_{1}+b_{2}, b_{3}\right) \\
& =\sigma\left(c+b_{1}, b_{2}\right) \sigma\left(c, b_{1}\right) \sigma\left(c+b_{1}+b_{2}, b_{3}\right) \\
& =\sigma\left(c+b_{1}, b_{2}+b_{3}\right) \sigma\left(b_{2}, b_{3}\right) \sigma\left(c, b_{1}\right) \\
& =\sigma\left(c, b_{1}+b_{2}+b_{3}\right) \sigma\left(b_{1}, b_{2}+b_{3}\right) \sigma\left(b_{2}, b_{3}\right)=\tilde{Y} .
\end{aligned}
$$

3. $s_{1}+s_{2}<p, s_{2}+s_{3}=p+t$ (hence $s_{1}+t<p$ ).

$$
\begin{aligned}
\tilde{X} \sigma\left(b_{1}, c\right) & =\sigma\left(b_{1}, b_{2}\right) \sigma\left(c, b_{1}+b_{2}+b_{3}\right) \sigma\left(b_{1}+b_{2}, b_{3}\right) \sigma\left(b_{1}, c\right) \\
& =\sigma\left(c, b_{1}+b_{2}+b_{3}\right) \sigma\left(b_{1}, b_{2}+b_{3}\right) \sigma\left(b_{2}, b_{3}\right) \sigma\left(b_{1}, c\right) \\
& =\sigma\left(b_{1}, c\right) \sigma\left(c+b_{1}, b_{2}+b_{3}\right) \sigma\left(c, b_{1}\right) \sigma\left(b_{2}, b_{3}\right) \\
& =\sigma\left(b_{1}, c+b_{2}+b_{3}\right) \sigma\left(c, b_{2}+b_{3}\right) \sigma\left(b_{2}, b_{3}\right) \sigma\left(c, b_{1}\right) \\
& =\tilde{Y} \sigma\left(c, b_{1}\right) .
\end{aligned}
$$

4. $s_{1}+s_{2}<p, s_{2}+s_{3}<p, s_{1}+s_{2}+s_{3} \geq p$

$$
\begin{aligned}
\tilde{X} & =\sigma\left(b_{1}, b_{2}\right) \sigma\left(c, b_{1}+b_{2}+b_{3}\right) \sigma\left(b_{1}+b_{2}, b_{3}\right) \\
& =\sigma\left(b_{1}, b_{2}+b_{3}\right) \sigma\left(b_{2}, b_{3}\right) \sigma\left(c, b_{1}+b_{2}+b_{3}\right)=\tilde{Y} .
\end{aligned}
$$

5. $s_{1}+s_{2}=p+r, s_{2}+s_{3}=p+t$. Thus $r+s_{3}=t+s_{1}$. Hence either both $r+s_{3}<p$ and $s_{1}+t<p$ or $r+s_{3} \geq p$ and $s_{1}+t \geq p$. The latter case differs from the former one only by the factor $\sigma\left(c, c+b_{1}+b_{2}+b_{3}\right)$ on both sides. So we consider only the former one. As in 3 above, we have to compare $\tilde{X} \sigma\left(b_{1}, c\right)$ and $\tilde{Y} \sigma\left(c, b_{1}\right)$. We have

$$
\begin{aligned}
\tilde{X} \sigma\left(b_{1}, c\right) & =\sigma\left(c, b_{1}+b_{2}\right) \sigma\left(b_{1}, b_{2}\right) \sigma\left(c+b_{1}+b_{2}, b_{3}\right) \sigma\left(b_{1}, c\right) \\
& =\sigma\left(c, b_{1}+b_{2}+b_{3}\right) \sigma\left(b_{1}+b_{2}, b_{3}\right) \sigma\left(b_{1}, b_{2}\right) \sigma\left(b_{1}, c\right) \\
& =\sigma\left(c, b_{1}+b_{2}+b_{3}\right) \sigma\left(b_{1}, b_{2}+b_{3}\right) \sigma\left(b_{2}, b_{3}\right) \sigma\left(b_{1}, c\right) \\
& =\sigma\left(c+b_{1}, b_{2}+b_{3}\right) \sigma\left(c, b_{1}\right) \sigma\left(b_{2}, b_{3}\right) \sigma\left(b_{1}, c\right) \\
& =\sigma\left(b_{1}, c+b_{2}+b_{3}\right) \sigma\left(c, b_{2}+b_{3}\right) \sigma\left(b_{2}, b_{3}\right) \sigma\left(b_{1}, c\right) \\
& =\tilde{Y} \sigma\left(c, b_{1}\right) .
\end{aligned}
$$

Thus the verification of $\sigma$ being a 2-cocycle is complete showing that $(A, \sigma)$ is an element of $\mathcal{S}$ strictly exceeding $\left(B, \sigma_{0}\right)$; this is a contradiction.

Proposition 2.4. If $A$ is a $G$-graded associative algebra and $[A]_{\varepsilon}$ the respective $(G, \varepsilon)$-Lie-superalgebra given by (5), then $A^{\sigma}$ with multipication

$$
(a b)^{\sigma}=\sigma(a, b) a b, \quad a \in A_{g}, \quad b \in A_{h},
$$


is a G-graded associative algebra and we have

$$
\left[A^{\sigma}\right]_{\varepsilon^{\prime}}=\left([A]_{\varepsilon}\right)^{\sigma}
$$

where $\sigma, \delta$, and $\varepsilon^{\prime}=\varepsilon \delta$ are as described before 2.2.

Proof. This is a simple computation. Take homogeneous $a, b \in A$ and compare the $\varepsilon^{\prime}$-bracket in $A^{\sigma}$ and the $\sigma$-twisted $\varepsilon$-bracket in $[A]$. That is,

$$
\begin{aligned}
{[a, b]_{\varepsilon^{\prime}} } & =(a b)^{\sigma}-\varepsilon^{\prime}(a, b)(b a)^{\sigma} \\
& =\sigma(a, b) a b-\varepsilon^{\prime}(a, b) \sigma(b, a) b a \\
& =\sigma(a, b)\left(a b-\left(\varepsilon^{\prime} \delta^{-1}\right)(a, b) b a\right) \\
& =\sigma(a, b)(a b-\varepsilon(a, b) b a) \\
& =\sigma(a, b)[a, b]_{\varepsilon}=\left([a, b]_{\varepsilon}\right)^{\sigma},
\end{aligned}
$$

proving our claim.

Example 2.5. As an application of Proposition 2.4, we are able to explain more clearly what is happening in the Weyl algebra examples considered in [Mo], Section 6.

In that paper the $n$-th Weyl algebra $\mathbb{A}_{n}=B_{1} \otimes \cdots \otimes B_{n}, B_{i} \cong \mathbb{A}_{1}, i=1, \ldots, n$, is considered first as an ordinary Lie superalgebra $L=L_{0} \oplus L_{1}$ with grading given by total degrees of elements and with the standard commutation factor $\varepsilon_{0}$ on $\mathbb{Z}_{2}$. If we use the product $\varepsilon_{0}^{n}$ of commutation factors $\varepsilon_{0}$ in each $B_{i}$, then we get a $\left(\mathbb{Z}_{2}^{n}, \varepsilon_{0}^{n}\right)$ Lie-superalgebra $L^{\prime}$. Now if we apply Scheunert's procedure to $L^{\prime}$, we arrive at an ordinary Lie superalgebra $L^{\prime \prime}=\left(L^{\prime}\right)^{\sigma}$. It is shown in [Mo] that $L$ is not isomorphic to $L^{\prime \prime}$ and in fact that the Lie product in $L^{\prime \prime}$ cannot be obtained from the associative structure of $\mathbb{A}_{n}$ as a twisted commutator in any way. However, Proposition 2.4 shows that $L^{\prime \prime}$ is the Lie superalgebra obtained from the usual superbracket on the associative algebra $\mathbb{A}_{n}^{\sigma}$. It is not difficult to see that $\mathbb{A}_{n}^{\sigma}$ is the twisted tensor product $\hat{\mathbb{A}}_{n}=B_{1} \hat{\otimes} \cdots \hat{\otimes} B_{n}$, where in $B \hat{\otimes} C,(b \otimes c) \cdot\left(b^{\prime} \otimes c^{\prime}\right)=(-1)^{|c|\left|b^{\prime}\right|} b b^{\prime} \otimes c c^{\prime}$ for $b, b^{\prime}, c, c^{\prime}$ homogeneous elements.

We close this section with some general results about graded algebras; we do not require that $G$ be abelian.

Lemma 2.6. Let $A$ be a $G$-graded associative algebra over a field $k$, with $G$ finite, and let $\sigma \in Z^{2}\left(G, k^{*}\right)$. Let $A^{\sigma}$ be the (associative) algebra with multiplication

$$
(a b)^{\sigma}:=\sigma(a, b) a b
$$

for $a, b$ homogeneous. Then

1. A is a PI-algebra if and only if $A^{\sigma}$ is a PI-algebra;

2. A is semiprime if and only if $A^{\sigma}$ is semiprime, provided $|G|^{-1} \in k$;

3. $A$ is semiprimitive if and only if $A^{\sigma}$ is semiprimitive, provided $|G|^{-1} \in k$.

Proof. First note that $A^{\sigma}$ is also $G$-graded, with $\left(A^{\sigma}\right)_{g}=A_{g}$ as vector spaces, and that since $\sigma(e, g)=1, e$ the identity element of $G$, it is clear that $A_{e} \cong\left(A^{\sigma}\right)_{e}$.

1. If $A$ is a PI-algebra, then so is $A_{e}$ and $\left(A^{\sigma}\right)_{e}$. Applying a theorem of Bergen and Cohen [BeC] (for a different proof holding even if $G$ is an infinite group, and $A$ is a Lie algebra; see [BZ]), it follows that $A^{\sigma}$ is a PI-algebra. Similarly the converse holds.

2. Assume that $A$ is semiprime. By [CM], Cor. 5.4, $N\left(A_{e}\right)=N(A) \cap A_{e}$, where $N(A)$ is the prime radical of $A$. Since $N(A)=\{0\}$ also $N\left(A_{e}\right)=$ $\{0\}=N\left(A^{\sigma}\right)_{e}$. Now by a theorem of $[\mathrm{CR}]$ we must have $N\left(A^{\sigma}\right)$ nilpotent. 
Since $A$ has no $|G|$-torsion, $N\left(A^{\sigma}\right)$ is a graded ideal by $[\mathrm{CM}]$, Cor. 5.5. But then $\left(N\left(A^{\sigma}\right)\right)^{\sigma^{-1}}$ is a nilpotent graded ideal of $A$, a contradiction, unless $N\left(A^{\sigma}\right)=\{0\}$.

3. The proof is very similar to that of 2 : replace the prime radical by the Jacobson radical $J(A)$ and $[\mathrm{CM}]$, Cor. 5.4 and 5.5, by [CM], Cor 4.2 and Theorem $4.4(3)$.

\section{LIE SUPERALGEBRAS WITHOUT ODD ELEMENTS}

In this section we prove Theorem 1.4 and Theorem 1.3.

Proof of Theorem 1.4. We first assume that $L$ is perfect. Without any loss of generality suppose that $k$ is algebraically closed (otherwise apply standard procedures of extending the ground field of coefficients). Using Proposition 2.4 of Section 2, we find $\sigma \in Z^{2}\left(G, k^{*}\right)$ such that in $A^{\sigma}$, the ordinary bracket $[a, b]=a b-b a$ differs from the $\varepsilon$-bracket $[a, b]=a b-\varepsilon(a, b) b a$ on homogeneous $a, b$ by a nonzero scalar $\sigma(a, b)$. So the same vector space $L$ under the new multiplication of $A$ becomes a Lie algebra under the ordinary bracket. Since $[a, b]^{\sigma}=\sigma(a, b)[a, b], \sigma(a, b) \in k^{*}$, the span of all $[a, b]^{\sigma}$ is the same as that of all $[a, b]$. So we have $L^{\sigma}=\left[L^{\sigma}, L^{\sigma}\right]^{\sigma}$. Similarly we observe that $A^{\sigma}$ is generated by $L^{\sigma}$ as an associative algebra. By Lemma 2.6, part $1, A^{\sigma}$ is a PI-algebra.

By Theorem 1.2 it follows that $\operatorname{dim} A^{\sigma}<\infty$; hence $\operatorname{dim} A<\infty$, as required.

Conversely, we now prove that the condition $L=[L, L]$ is not only sufficient but also necessary for having any PI-envelope finite-dimensional.

We will show that if $L \neq[L, L]$, then $L$ has a PI-envelope which is not finitedimensional. First consider the case when $L$ is an ordinary (graded) Lie algebra. Let $M=L /[L, L]$. The quotient map $\phi: L \rightarrow M$ induces a homomorphism of associative algebras

$$
\psi: U(L) \rightarrow U(M) \cong k\left[X_{1}, \ldots, X_{s}\right], s=\operatorname{dim} M
$$

Let $\chi: L \rightarrow \operatorname{End}(V)$ be any faithful finite-dimensional representation of $L$; such exists by the extension of Ado's Theorem obtained by Scheunert [S79a]. Letting $\tau:=\psi \otimes \chi$, the Lie tensor product of these maps, we obtain a homomorphism of $L$ into $Q=U(M) \otimes \operatorname{End}(V)$.

Now $\tau$ is an imbedding since $\chi$ is faithful, and it is not difficult to see that $A:=\tau(U(L))$ is infinite-dimensional (since if $X_{i}=\psi\left(x_{i}\right)$, the images under $\tau$ of the distinct ordered monomials in the $x_{i}$ are linearly independent). Moreover $Q$ is a PI-algebra, since $U(M)$ is commutative and $\operatorname{End}(V)$ is finite-dimensional. Thus $A \subset Q$ is an infinite-dimensional PI-envelope of $L$.

Finally if $\varepsilon$ is non-trivial, we may use Scheunert's construction as before and pass to $L^{\sigma}$, an ordinary Lie algebra. If $A$ is the PI-envelope constructed as above, then $A^{\sigma-1}$ is an infinite-dimensional PI-envelope of $L$ (where we have used Lemma 2.6 for PI as above).

Remark 3.1. Applying M. Scheunert's procedure enables us to generalize V. Kac's Theorem [Kac] about solvability of Lie superalgebras to general $(G, \varepsilon)$-Lie-superalgebras and to show that a $(G, \varepsilon)$-Lie-superalgebra $L=L_{+} \oplus L_{-}$is solvable iff $L_{+}$ is solvable. 
We recall that a $(G, \varepsilon)$-Lie-superalgebra $L$ is called semisimple if it has no $G$ graded solvable ideals.

Before we pass to the proof of Theorem 1.3 we need the following auxiliary result.

Proposition 3.2. Let $L$ be a $G$-graded Lie algebra, $G$ a finite abelian group. If $L$ is graded semisimple, then it is semisimple in the ordinary sense.

Proof. We start with the case where $k$ is an algebraically closed field. Suppose $L$ has a solvable ideal $M$ which is not graded. Consider the dual group $\hat{G}$. Then $\hat{G}$ acts on $L$ by automorphisms in a natural way: if $\chi \in \hat{G}, x \in L_{g}$, then $\chi * x=\chi(g) x$. An ideal of $L$ is $G$-graded if and only if it is $\hat{G}$-invariant. Now for any $\chi \in \hat{G}$ we have that $\chi * M$ is an ideal of $L$ and thus that $\tilde{M}=\sum_{\chi \in \hat{G}} \chi * M \supset N$ is a graded ideal. Since the sum of two solvable ideals is always solvable, we conclude that $\tilde{M}$ is a non-trivial $G$-graded solvable ideal of $L$. Hence $\tilde{M}=\{0\}$ and then $M=\{0\}$, as stated.

If $k$ is not algebraically closed, then the standard procedure of extending the field of coefficients works because then the extended algebra is (graded) semisimple (respectively, solvable) if and only if this is true for the original algebra.

Proof of Theorem 1.3. Let $\sigma \in Z^{2}\left(G, k^{*}\right)$ be a 2-cocycle such that $L^{\sigma}$ is an ordinary Lie algebra and $A^{\sigma}$ is a PI-envelope of $L^{\sigma}$, as in the proof of Theorem 1.4. Obviously $L^{\sigma}$ is a $G$-graded algebra without $G$-graded solvable ideals. It follows by Proposition 3.2 that $L^{\sigma}$ is an ordinary semisimple Lie algebra, hence by Theorem 1.1 we have that $A^{\sigma}$, as a PI-envelope of $L^{\sigma}$, is finite-dimensional and Jacobson semisimple. Now we have shown that $A$ is a finite-dimensional algebra. By Lemma 2.6, part 3, $A$ is also semisimple, as required.

\section{General CASE AND CONCLUding REMARKS}

In this section we will prove Theorems 1.5 and 1.6. The proof of finite-dimensionality of PI-envelopes of certain $(G, \varepsilon)$-Lie-superalgebras is greatly facilitated by the following argument.

Lemma 4.1. Suppose $L$ is a $(G, \varepsilon)$-Lie-superalgebra such that any PI-envelope of $L_{+}$is finite-dimensional. Then any PI-envelope of $L$ is finite-dimensional as well.

Proof. This is a simple consequence of our statement of the PBW-Theorem (Theorem 2.1). If $A$ is a PI-envelope of $L$, then $A$ is a homomorphic image of $U(L)$ under a homomorphism $\varphi: U(L) \rightarrow A$. If $T=\varphi\left(U\left(L_{0}\right)\right)$, then $T$ is a PI-envelope of $L_{0}$, hence finite-dimensional. Now by the PBW-Theorem, if $\left\{e_{1}, \ldots, e_{n}\right\}$ is a basis for $L_{-}$, then the whole of $A$ is the span of all elements of the form $t \varphi\left(e_{i_{1}}\right) \cdots \varphi\left(e_{i_{k}}\right), 1 \leq$ $i_{1}<i_{2}<\cdots<i_{k} \leq n, t \in T$. It follows then that $A$ is also finite-dimensional.

Proof of Theorem 1.5. In all such cases $L_{0}\left(=L_{+}\right)$is semisimple unless $L$ is of Cartan type in which case $L_{0}$ is perfect. Then Theorem 1.4 applies and any PIenvelope of $L_{0}$ is finite-dimensional. Now Lemma 4.1 applies yielding the finitedimensionality of any PI-envelope of $L$.

Now we want to show that Theorem 1.2 fails not only for perfect Lie superalgebras but also for certain simple Lie superalgebras.

Theorem 4.2. Any of the simple Lie superalgebras $L=\operatorname{sl}(n, m), n, m \geq 1, n \neq m$, or $W(n), n \geq 2$, has an infinite-dimensional PI-envelope. 
Proof. The main reason for having such an envelope is having infinitely many pairwise non-isomorphic representation of the same dimension. Both of these types of algebras have highest weight modules [Kac] with highest weights of the form $\lambda z^{*}, \lambda \in k$, where $g+k z$ is the maximal reductive subalgebra of $L_{0}$. The corresponding Verma module $V(\lambda)$ for such a weight has dimension $t=2^{\operatorname{dim} L_{1}}$ (again one applies the PBW-Theorem and the construction of $V(\lambda)$ as induced from a 1-dimensional representation of a "Borel" subalgebra). Take the irreducible highest weight module $L(\lambda)$. Let $\rho_{\lambda}$ be a representation of $L$ in $L(\lambda)$. Consider then $\rho=\bigoplus_{\lambda \in k^{*}} \rho_{\lambda}$. The image $\rho_{\lambda}(L)$ of $L$ in each of these representations generates an associative algebra satisfying a standard polynomial identity $S_{2 t}=0$.

It remains to show that the associative algebra $A=\langle\rho(L)\rangle$ generated by $\rho(L)$ cannot be finite-dimensional. If $\operatorname{dim} A<n$, then there exist $\alpha_{0}, \alpha_{1}, \cdots, \alpha_{n} \in k$, not all 0 , such that $\alpha_{0}+\alpha_{1} \rho(z)+\cdots+\alpha_{n} \rho(z)^{n}=0$. Then we have also

$$
\alpha_{0} \cdot 1_{L(x)}+\alpha_{1} \rho_{\lambda}(z)+\cdots+\alpha_{n} \rho_{\lambda}(z)^{n}=0
$$

i.e., all $\lambda \in k^{*}$ satisfy a polynomial equation of degree $n$ since $\rho_{\lambda}(z)=z \cdot 1_{L(\lambda)}$. This is obviously impossible and thus the proof is complete.

Remark 4.3. This is an appropriate place to give a more instructive proof of Theorem 1.1 from [Ba 85].

There are two steps in proving that theorem in [Ba 85]: the first is that any semiprimitive PI-envelope of a semisimple Lie algebra is finite-dimensional and the second is that the Jacobson radical of an arbitrary PI-envelope is zero. We want to modify here the proof of the first step.

First of all, before [Ba 85] was written M. Duflo had already determined the structure of primitive ideals of the universal enveloping algebra of a finite-dimensional semisimple Lie algebra $L$ over an algebraically closed field of characteristic zero. By Duflo's theorem [Du], any of these ideals have the form $J(\lambda)=$ $\operatorname{Ann}_{U(L)} L(\lambda)$ of the annihilator of an irreducible highest weight module $L(\lambda)$ with highest weight $\lambda$. Now if $A$ is a semiprimitive PI-envelope of $L$, and $A$ satisfies a polynomial identity of degree $d$, then $A=U(L) / P$ and any $J(\lambda) \subset P$ whenever $\operatorname{dim} L(\lambda)>\frac{d}{2}$. It follows from the dimension formula for $L(\lambda)$ that there exist only finitely many $\lambda$ with $\operatorname{dim} L(\lambda) \leq n$ for any natural number $n$, hence $P$ is the intesection of only finitely many ideals of finite codimension, proving $\operatorname{dim} A<\infty$.

Yet another proof of the finite-dimensionality of $A$ can be given by using a paper of Farkas [Fa] where the author proves that if $R$ is a finitely generated semiprime PI-algebra over a field $F$ (not necessarily of characteristic zero) and every finitedimensional $R$-module is semisimple (which is obviously the case in our situation), then $R$ is finite-dimensional. This result on PI-algebras was used to give a purely algebraic (and very short) proof of a theorem attributed to Weil: if every finitedimensional representation of a finitely generated group $\Gamma$ is semisimple, then there are only finitely many isomorphism classes of $\Gamma$-representations for each degree.

Proof of Theorem 1.6. $1 \Rightarrow 3$. As was shown in Theorem 1.4, if $L_{+}$is not perfect, then there is an infinite-dimensional PI-envelope $A$ of $L_{+}$. Let $\rho: U\left(L_{+}\right) \rightarrow A$ be the natural surjective homomorphism. Then $A$ becomes a $U\left(L_{+}\right)$module via $u * a=\rho(u) a, u \in U\left(L_{+}\right), a \in A$. Of course, $U\left(L_{+}\right)$is a left regular $U\left(L_{+}\right)$-module also. Since $U(L)$ is also a left $U\left(L_{+}\right)$-module we can form the induced $U(L)$ module (cf. [Bl]) $P=U(L) \otimes_{U\left(L_{+}\right)} A$. This is a left $U(L)$ and right $A$-module. 
Let $\tau: U(L) \rightarrow \operatorname{End}(P)$ be the respective homomorphism. Now $\operatorname{dim} A=\infty$ and $1 \otimes 1$ generates the whole of $P$ under the left $U(L)$-action: $1 \otimes A$ is generated by the action of $U\left(L_{+}\right)$and then the action of $U(L)$ generates the whole of $P$. Thus it is obvious that the image of $U(L)$ in $\operatorname{End}(P)$ is an infinite-dimensional algebra. Now the left action of $U(L)$ on $P$ commutes with the right action of $A$. Under this latter action $P$ is a finitely generated $A$-module of rank $\leq 2^{\operatorname{dim} L_{-}}$. Thus the image of $U(L)$ under $\tau$ belongs to the endomorphism ring of a finitely generated module over a PI-algebra, hence is a PI algebra itself. Thus this image $\tau(U(L))$ is an infinite-dimensional PI-envelope of $U(L)$.

$1 \Leftrightarrow 2$. If any graded PI-homomorphic image of $U(L)$ is finite-dimensional, then any PI-envelope is finite-dimensional because this is also a graded homomorphic image of $U(L)$. Now if any PI-envelope is finite-dimensional and $I$ is a $G$-graded ideal of $U(L)$ such that $U(L) / I$ is PI, then we can take a faithful finite-dimensional representation $\chi: L \rightarrow \operatorname{End} V$ of the $(G, \varepsilon)$-superalgebra $L$, as in the proof of Theorem 1.4. We then form a $G$-graded associative algebra $B=U(L) / I \oplus$ End $V$. It is obvious that $B$ is a PI-algebra. The natural homomorphism $\rho$ of $L$ into $B$ sending $x \in L$ into $(x+I, \chi(x))$ is faithful and $\rho(L)$ generates an associative algebra $A$ which is a PI-envelope of $L$. By our hypothesis $A$ is finite-dimensional and its projection onto the first component is $U(L) / I$. So this latter is finite-dimensional, as required.

$3 \Rightarrow 1$. This is an easy combination of Theorem 1.4 and Lemma 4.1.

Remark 4.4. We do not know the structure of the PI-envelopes of semisimple ordinary Lie superalgebras.

Remark 4.5. It would be interesting to find the structure of the PI-envelope of $L$ in the case when $L_{+}$is semisimple.

\section{REFERENCES}

[Ba 85] Yu. A. Bahturin, On the structure of the PI-envelope of a finite-dimensional Lie algebra, Soviet Math. (Iz. VUZ) 29 (1985) no. 11, 83-87.

[BMPZ ] Yu. A. Bahturin, A. Mikhalev, V. Petrogradskii, M. Zaicev, Infinite Dimensional Lie Superalgebras, Expos. Math. vol 7, Walter de Gruyter, Berlin, 1992. MR 94b:17001

[BeC] J. Bergen and M. Cohen, Actions of commutative Hopf algebras, Bull. LMS 18 (1986), 159-164. MR 87e:16052

[Bi] Yuly Billig, On the homomorphic image of a special Lie algebra, Mat. Sc 136 (178)(1988), 320-323; English transl. in Math. USSR Sb. 64 (1989) MR 89k:17015

[Bl] R. J. Blattner, Induced and produced representations of Lie algebras, Transactions AMS 144 (1969), 457-474. MR 46i:7338a

[BZ] Y. Bahturin, M.Zaicev, Identities of graded Lie algebras, J. Algebra 205 (1998), 1-12. CMP 98:15

[CM] M. Cohen and S. Montgomery, Group-graded rings, smash products, and group actions, Trans. AMS 282 (1984), 237-258. MR 85i: 16002

[CR] M. Cohen and L. Rowen, Group graded rings, Comm. Algebra 11 (1983), 1253 -1270. MR 85b: 16002

[Du] M. Duflo, Sur la classification des ideaux primitifs dans l'algebre enveloppante d'une algebre de Lie semi-simple, Ann. Math. 105 (1977), 107-120. MR 55:3013

[Fa] D. Farkas, Semisimple representations and affine rings, Proc. AMS 101 (1987), 237 238. MR 88h: 16027

[Kac] V. Kac, Lie superalgebras, Advances in Math. 26 (1977), 8-96. MR 58:5803

[Mo] S. Montgomery, Constructing simple Lie superalgebras from associative graded algebras, J. Algebra 195 (1997), 558 - 579. CMP 98:01

[Po] H. Pop, A generalization of Scheunert's theorem on cocycle twisting of Lie color algebras, preprint, q-alg 9703002. 
[S79a] M. Scheunert, Generalized Lie algebras, J. Math Physics 20 (1979), 712-720. MR 80f: 17007

[S79b] M. Scheunert, The Theory of Lie Superalgebras, Lecture Notes in Math., vol. 716, Springer-Verlag, Berlin, 1979. MR 80i:17005

Department of Algebra, Moscow State University, 119899 Moscow, Russia

E-mail address: bahturin@mech.math.msu.su

Department of Mathematics, University of Southern California, Los Angeles, CalIFORNIA 90089-1113

E-mail address: smontgom@math.usc.edu 